\title{
GESTIÓN DEL CONOCIMIENTO, LIDERAZGO, DISEÑO E IMPLEMENTACIÓN DE LA ESTRATEGIA: UN ESTUDIO EMPÍRICO EN PEQUEÑAS Y MEDIANAS EMPRESAS
}

\author{
KNOWLEDGE MANAGEMENT, LEADERSHIP, STRATEGY DESING AND \\ IMPLEMENTATION: AN EMPIRICAL STUDY IN SMALL AND MEDIUM-SIZED FIRMS
}

\author{
Emilio Rodríguez-Ponce ${ }^{1}$ \\ Liliana Pedraja-Rejas ${ }^{2}$ \\ Milagros Delgado 3 \\ Recibido 9 de julio de 2009, aceptado 7 de octubre de 2010 \\ Received: July 9, 2009 Accepted: October 7, 2010
}

Juan Rodríguez-Ponce ${ }^{4}$

\begin{abstract}
RESUMEN
El presente estudio tiene por objetivo identificar la existencia de relaciones significativas entre la gestión del conocimiento, los diferentes estilos de liderazgo y el diseño e implementación de la estrategia. La investigación se llevó a cabo con 78 altos directivos de pequeñas y medianas empresas que operan en el norte de Chile. Los resultados demuestran que los estilos de liderazgo transformacional y transaccional impactan positiva y significativamente sobre la calidad del diseño de la estrategia. Otro hallazgo relevante está relacionado con que la creación y aplicación de conocimiento es un determinante significativo de la calidad de la estrategia y a su vez el diseño de la estrategia es un determinante clave de la implementación de la estrategia. Por lo tanto, los estilos de liderazgo como la gestión del conocimiento son relevantes para lograr mejores niveles de diseño e implementación de la estrategia.
\end{abstract}

Palabras clave: Gestión del conocimiento, liderazgo, diseño e implementación de la estrategia.

\begin{abstract}
This study has the aim to identify the existence of significant relations between knowledge management, leadership styles and the strategy design and implementation. The research works with a sample of 78 top managers of small and mediumsized firms in northern Chile. The results show that the transformational and transactional leadership have a positive impact on the strategy design quality. In addition, the knowledge creation and application are a significant determinant in the strategy quality; as well the strategy design is a decisive determinant to the strategy implementation. Therefore, the leadership styles and the knowledge management are relevant to achieve better results in the strategy design and implementation in small and medium-sized firms.
\end{abstract}

Keywords: Knowledge management, leadership, strategy design and implementation.

\section{INTRODUCCIÓN}

En los tiempos que corren el conocimiento se ha convertido en un recurso estratégico. En la sociedad del conocimiento la creación efectiva, uso y difusión del conocimiento es la clave del éxito en las organizaciones. En consecuencia, estas tienen un nivel superior de conocimiento, son capaces de coordinar y combinar sus recursos tradicionales en nuevos y distintos caminos, creando un valor diferenciador para sus clientes.

La gestión del conocimiento tiene impacto sobre la generación de nuevos productos, la disminución de costos, la generación de nuevas oportunidades de negocios. Sin embargo, siendo todos estos aspectos de carácter estratégico, recientemente se ha demostrado que la gestión del conocimiento puede influir en el diseño e implementación de la estrategia [1 y 2].

Las acciones de crear, compartir y aplicar conocimientos permiten diseñar decisiones más racionales, flexibles y con mayor contraste de ideas, todo lo cual favorece el diseño de las decisiones estratégicas [3 y 4]. Más aún, el acto de compartir conocimiento entre el equipo de alta dirección conllevará a decisiones que generen mayor compromiso de los participantes en su implementación [5-6].

1 Instituto de Alta Investigación. Universidad de Tarapacá. 18 de Septiembre 2222 Arica-Chile. E-mail: erodrigu@uta.cl

2 Escuela Ingeniería Industrial, Informática y de Sistemas. Universidad de Tarapacá. 18 de Septiembre 2222 Arica-Chile. E-mail: 1pedraja@ uta.cl

3 Comisión Nacional de Acreditación. Ricardo Lyon 1532, Santiago-Chile. E-mail: mdelgado@cnachile.cl

4 Universidad de Tarapacá. CFT Tarapacá. Avda. Gral. Velásquez 1775, Arica-Chile. E-mail: jrodrigup@uta.cl 
Por otra parte, recientes investigaciones han demostrado la importancia de los estilos de liderazgo para explicar el éxito en las organizaciones [7], así como su influencia sobre el proceso de toma de decisiones estratégicas [8] y, más directamente, sobre el diseño e implementación de la estrategia [1]

Esta investigación pretende indagar en la relación de los estilos de liderazgo y la gestión del conocimiento con el diseño e implementación de la estrategia. Para tal efecto se realiza una revisión teórica de la bibliografía básica sobre estos temas, para luego plantear las hipótesis correspondientes. Posteriormente se presenta la metodología de la investigación, que se sustenta en un estudio empírico que considera una muestra de 78 altos directivos de pequeñas y medianas empresas que operan en el norte de Chile, se analizan los resultados y se establecen conclusiones, alcances e implicaciones. Entre los resultados más relevantes del estudio se tiene que efectivamente los estilos y la gestión del conocimiento son variables relevantes para el diseño y la posterior implementación de la estrategia.

Este trabajo es una contribución al estado del arte ya que analiza el impacto conjunto de los estilos de liderazgo y de la gestión del conocimiento sobre el diseño e implementación de la estrategia. La mayoría de las investigaciones sobre estas temáticas estudian las relaciones individuales de estas variables. Conjuntamente, el estudio de campo considera empresas pequeñas y medianas de Chile, lo cual es otro aspecto relevante ya que la mayoría de los estudios de campo referenciados en la literatura internacional se realizan en países desarrollados, siendo por tanto esta investigación una excepción en relación con conseguir evidencia empírica sobre empresas que operan en un país en vías de desarrollo.

\section{ANÁLISIS TEÓRICO}

\section{La gestión del conocimiento}

Nonaka y Takeuchi [9] sostienen que el conocimiento nuevo en las organizaciones surge de los individuos, pero en el proceso de compartirlo es transformado en un conocimiento valioso para la organización como un todo y, por lo tanto, es posible establecer cuatro patrones para la creación de conocimiento en cualquier organización:

- De tácito a tácito, que se produce cuando los individuos comparten conocimiento mediante observación, imitación y/o práctica, convirtiéndolo en su propio conocimiento tácito; sin embargo, ninguno de los individuos obtiene una visión sistemática en su conocimiento, y el mismo no puede ser utilizado por la organización como explícito.

- De explícito a explícito, que se produce cuando un individuo combina elementos del conocimiento existente, adaptando o mostrando una nueva perspectiva del todo; sin embargo, esta forma de conocimiento no implica la expansión de la base de conocimiento de la organización.

- De tácito a explícito, que se produce cuando un individuo es capaz de articular los fundamentos de su conocimiento tácito, lo convierte en explícito y se logra por consiguiente un crecimiento de la base de conocimiento.

- De explícito a tácito, que se produce cuando el conocimiento explícito es compartido a lo largo de la organización, los demás comienzan a utilizarlo y por lo tanto lo amplían, extienden e incorporan en su propio conocimiento tácito.

Estos cuatro factores existen en una interacción dinámica que se convierte en una espiral de conocimiento, que crece cada vez más a alto nivel y por lo tanto lleva al crecimiento organizacional.

La gestión del conocimiento puede ser empleada en distintos tipos de organizaciones y es principalmente exitosa en aquellas en las cuales los diferentes niveles de la empresa tienen un aporte significativo a la firma [10]. En la nueva economía, la capacidad de las organizaciones para generar valor económico de su conocimiento es un elemento central para generar ventaja competitiva [11]. En efecto, tal y como lo plantean Alavi y Leidner [12], la gestión del conocimiento tiene como objetivo fundamental soportar la creación, la transferencia, y la aplicación del conocimiento en las organizaciones.

En este contexto, se puede sostener que el proceso de gestión del conocimiento incluye tres etapas [2], a saber:

Crear conocimiento, que implica exploración, combinación y el descubrimiento de conocimiento mediante el hacer. Los individuos al interior de una organización crean nuevos conocimientos mediante conexiones intuitivas de las ideas existentes o a través de la interacción con otros individuos de la organización.

Compartir conocimiento, que se produce cuando los individuos al interior de una organización transfieren y comparten el conocimiento. Al compartir este conocimiento, éste se incrementa y llega a ser más valioso, se producen 
sinergias que hacen que el total del conocimiento alcanzado sea mayor cuantitativa y cualitativamente que la suma de los conocimientos individuales.

Aplicar conocimiento, que implica convertir el conocimiento en un resultado valioso para la organización. La aplicación de conocimiento puede conducir a la generación de productos y servicios, pero también a la generación de ideas que llevan a una mejor toma de decisiones estratégicas.

\section{El diseño e implementación de la estrategia}

La estrategia presenta dos partes esenciales: el diseño y la implementación. El diseño o formulación de la estrategia es un proceso colectivo que se sustenta en el desarrollo y aplicación de competencias distintivas, difíciles de imitar [13]. El diseño ha sido ampliamente estudiado y es posible sostener que a partir de la definición de la misión y los valores corporativos, el diseño mismo de la estrategia considera un análisis de las fuerzas competitivas, y de los recursos y capacidades para llegar a la definición de la estrategia corporativa y sus consecuentes estrategias funcionales. El proceso que conduce a la elección de la estrategia de la empresa es un proceso de toma de decisiones; dicho proceso se nutre por la calidad de la gestión del conocimiento que distingue a la organización [1].

Las empresas adoptan sus decisiones estratégicas, pero más del 50\% de dichas decisiones fracasan. En consecuencia, la implementación de la estrategia no es una tarea menor, ya que tiene una implicación estructural en los niveles de éxito y fracaso que se alcancen finalmente. La implementación de la estrategia tiene dos categorías fundamentales: 1) la dirección y administración, que incluye la cultura organizativa; y 2) la implementación operacional, que considera apoyo administrativo, recompensas, mejores prácticas y presupuesto [14].

\section{Liderazgo}

El estilo de liderazgo se relaciona con la eficacia en empresas pequeñas, medianas y grandes, y en organizaciones con fines de lucro y sin fines lucro [5, 15 y 16]. El estilo de liderazgo influye sobre los procesos, los productos, los servicios y, consecuentemente, sobre los resultados de la firma organización [17].

La teoría de Bass [18] distingue tres estilos de liderazgo, el transformacional, el transaccional y el "laissez faire". Esta teoría establece que el líder transformacional guía a sus seguidores y los inspira, estableciendo desafíos y una motivación basada en el desarrollo personal de quienes lo siguen. En efecto, el líder transformacional conduce al logro de estándares de excelencia, individuales y colectivos, a través del establecimiento de una visión y una misión comunes. Por su parte, el liderazgo transaccional es aquel en el cual los seguidores se motivan en base a los beneficios esperados por el logro de las metas o tareas encomendadas. Ciertamente, el liderazgo transaccional implica un proceso de negociación entre el líder y los seguidores. Finalmente, el estilo "laissez faire" se caracteriza porque el líder renuncia al control y permite que sean los subordinados los que tomen las decisiones.

\section{Las pequeñas y medianas empresas}

La definición de pequeñas y medianas empresas tiene múltiples acepciones; sin embargo, en este artículo se maneja el criterio empleado en trabajos anteriores [1], que considera como pequeñas y medianas firmas aquellas que cuentan con entre 10 y 50 trabajadores. Este tipo de empresas tienen un conjunto de características que las diferencian de las grandes compañías.

Los propietarios en las pequeñas y medianas empresas desean preservar el control en todos sus ámbitos, por lo que adoptan las principales decisiones. Además, el factor tiempo es un aspecto crítico en estas decisiones, ya que deben responder a un entorno exigente y generalmente hostil [19]. En este contexto, en las pequeñas empresas las decisiones suelen ser adoptadas por una sola persona, quien generalmente, a su vez es el propietario. A medida que se incrementa el tamaño de la empresa suelen incorporarse nuevos miembros a la toma de decisiones. De igual modo, las relaciones entre directivo y empleados suele ser más informal en las pequeñas empresas, y el nivel de formalidad crece a medida que aumenta el tamaño de la firma [20].

En consecuencia, el tamaño de la firma influye en los procesos de toma de decisiones [21]. De hecho, las reacciones estratégicas son diferentes dependiendo del tamaño de la empresa [22]; la aplicación de fundamentos de dirección y gestión de empresas son más precarios en las pequeñas y medianas empresas, en comparación con empresas grandes que tienen sistemas de gestión consolidados. Por ende, es necesario establecer que los resultados de esta investigación se refieren específicamente a pequeñas y medianas empresas $y$, por lo tanto, resulta probable que en las grandes empresas, estos resultados no sean necesariamente los mismos o, al menos, requieren un análisis pormenorizado.

\section{EL MODELO DE ANÁLISIS}

La presente investigación procura identificar la existencia o no de relaciones estadísticamente significativas entre los estilos de liderazgo, la gestión del conocimiento y el diseño e implementación de la estrategia. 
La Figura 1 muestra el modelo general de análisis. En este sentido, las hipótesis a contrastar son las siguientes:

\section{H-1:}

Los estilos de liderazgo transformacional y transaccional, así como la creación de conocimiento, el compartir conocimiento y la aplicación de conocimiento, tienen un impacto estadísticamente significativo para explicar la calidad del diseño de la estrategia.

\section{H-2:}

Los estilos de liderazgo transformacional y transaccional, así como la creación de conocimiento, el compartir conocimiento y la aplicación de conocimiento, y la calidad del diseño de la estrategia tienen un impacto estadísticamente significativo para explicar la calidad de la implementación de la estrategia.

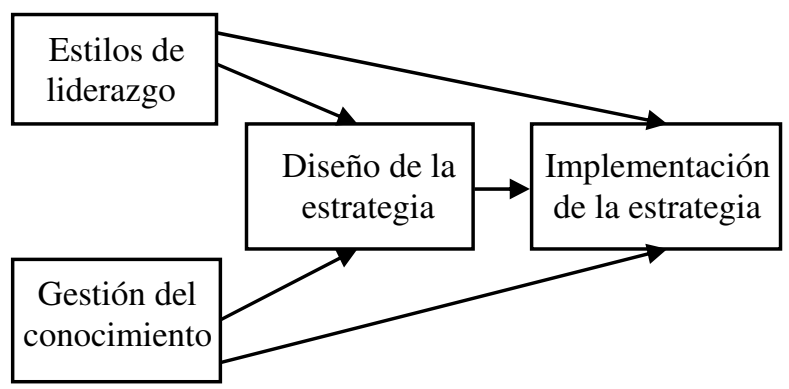

Figura 1. Modelo de análisis.

\section{METODOLOGÍA DE LA INVESTIGACIÓN}

\section{Muestra}

Se aplicó un cuestionario a 420 altos directivos de pequeñas y medianas empresas que operan en el norte de Chile. Se obtuvieron 78 respuestas, lo que corresponde a una tasa de respuesta del $18,6 \%$. Se insistió hasta tres veces con los altos directivos, en un período que se extendió por cinco meses.
El 60,25\% de estas empresas comercializan sus productos localmente, es decir, en el mercado de Chile; mientras el $25,64 \%$ de las empresas de la muestra comercializan sus productos en Perú y Bolivia; destacándose un 14,10\% de las firmas que operan en mercados internacionales tales como los Estados Unidos, Europa o Asia.

\section{Unidad y nivel de análisis}

La unidad de análisis estuvo constituida por la percepción de los estilos de liderazgo y la gestión del conocimiento por parte de los directivos de pequeñas y medianas empresas. El nivel de análisis con el que se trabajó es la alta dirección o ápice estratégico.

\section{Variables y medidas}

Se empleó un conjunto de variables y medidas validadas en investigaciones previas (Tabla 1) y se usó la escala de Likert (1-7). Para cada caso se midió la fiabilidad de las variables y medidas de acuerdo con el cálculo del alpha de Cronbach.

\section{Métodos}

Se utilizó un modelo de regresión múltiple que permitió evaluar la pertinencia de cada una de las hipótesis de la investigación. La expresión de dicho modelo se expresa en (1):

$$
V_{x}=A+a \beta_{1}+b \beta_{2}+c \beta_{3}+\ldots+n \beta_{n}+E_{i}
$$

donde $\mathrm{V}_{\mathrm{x}}$ : variable dependiente denominada eficacia; $\mathrm{A}$ : punto de intersección del modelo, que conceptualmente considera las variables omitidas por la ecuación de regresión; $a, b$, c: variables independientes; $\beta_{1}, \beta_{2}, \beta_{3} \ldots$, $B_{n}$ representan el factor de relación entre las variables independientes respectivas y la variable dependiente, y $\mathrm{E}_{\mathrm{i}}$ : valor que representa la parte del error de estimación que no es capturado por la ecuación de regresión. 
Tabla 1. Variables y medidas.

\begin{tabular}{|c|c|c|c|}
\hline Variables & Ítems & $\begin{array}{l}\text { Sustento } \\
\text { teórico }\end{array}$ & $\begin{array}{l}\text { Alpha de } \\
\text { Cronbach }\end{array}$ \\
\hline $\begin{array}{c}\text { Estilo } \\
\text { transformacional }\end{array}$ & $\begin{array}{l}\text { - } \quad \text { El líder comparte la misión y la visión con sus seguidores. } \\
\text { - } \quad \text { Los seguidores comparten y siguen con entusiasmo los objetivos } \\
\text { del líder. } \\
\text { - } \quad \text { Los seguidores comparten y siguen con entusiasmo la visión de } \\
\text { largo plazo del líder. } \\
\text { - } \quad \text { Los seguidores aceptan con entusiasmo los desafíos profesionales } \\
\text { que les plantea el líder. } \\
\text { - Los seguidores comparten y aceptan con entusiasmo el papel que } \\
\text { les corresponde jugar en la organización. }\end{array}$ & $\begin{array}{l}\text { Adaptado de } \\
\text { Bass y } \\
\text { Avolio [23] }\end{array}$ & 0,794 \\
\hline $\begin{array}{l}\text { Creación de } \\
\text { conocimiento }\end{array}$ & $\begin{array}{l}\text { - La institución tiene un eficiente sistema de exploración de } \\
\text { información interna y externa. } \\
\text { - La información obtenida por diversas fuentes es eficientemente } \\
\text { procesada e integrada al interior de la organización. } \\
\text { - La institución tiene un sistema que le permite identificar hallazgos } \\
\text { importantes para su quehacer tanto de fuentes internas como } \\
\text { externas. } \\
\text { - Los directivos de la institución crean nuevos conocimientos } \\
\text { considerando el sistema de exploración, detección de hallazgos } \\
\text { e integración de información. } \\
\text { - Los directivos de la institución interactúan entre sí favoreciendo } \\
\text { la creación de conocimiento. }\end{array}$ & $\begin{array}{l}\text { Basado } \\
\text { en March; } \\
\text { Nonaka y } \\
\text { Takeuchi } \\
\text { [24 y 9] }\end{array}$ & 0,738 \\
\hline $\begin{array}{l}\text { Compartir } \\
\text { conocimiento }\end{array}$ & $\begin{array}{l}\text { - Los directivos de la organización intercambian conocimientos } \\
\text { entre sí. } \\
\text { - Los directivos de la organización se transfieren conocimientos } \\
\text { mutuamente. } \\
\text { - Los directivos de la institución comparten conocimiento } \\
\text { mutuamente. } \\
\text { - Los directivos de la institución aplican los conocimientos generados } \\
\text { y compartidos. } \\
\text { - Los directivos toman decisiones basados en la aplicación de } \\
\text { conocimientos previamente generados. }\end{array}$ & $\begin{array}{l}\text { Basado en } \\
\text { Nonaka y } \\
\text { Takeuchi; } \\
\quad \text { Kim } \\
\quad \text { y } \\
\text { Maugborne } \\
\quad[9 \text { y 6] }\end{array}$ & 0,756 \\
\hline $\begin{array}{l}\text { Aplicación de } \\
\text { conocimiento }\end{array}$ & $\begin{array}{l}\text { - Los directivos de la institución aplican los conocimientos generados } \\
\text { y compartidos. } \\
\text { - Los directivos toman decisiones basados en la aplicación de } \\
\text { conocimientos previamente generados. }\end{array}$ & $\begin{array}{l}\text { Basado en } \\
\text { Nonaka y } \\
\text { Takeuchi [9] }\end{array}$ & 0,732 \\
\hline $\begin{array}{l}\text { Diseño de la } \\
\text { estrategia }\end{array}$ & $\begin{array}{l}\text { - } \quad \text { Estrategia adoptada fue de la máxima calidad. } \\
\text { - } \quad \text { La estrategia adoptada permite cumplir cabalmente los objetivos } \\
\text { perseguidos. } \\
\text { - } \quad \text { La estrategia adoptada presenta una solución eficaz y eficiente a } \\
\text { la problemática que se deseaba resolver. } \\
\text { - La estrategia adoptada crea valor para la institución, favorece su } \\
\text { desarrollo, y el cumplimiento de sus fines. }\end{array}$ & $\begin{array}{l}\text { Basado en } \\
\text { Amason; } \\
\text { Dean y } \\
\text { Sharfman } \\
\text { [25 y 26] }\end{array}$ & 0,688 \\
\hline $\begin{array}{l}\text { Implementación } \\
\text { de la estrategia }\end{array}$ & $\begin{array}{l}\text { - La estrategia fue implementada logrando altos niveles de compromiso } \\
\text { por parte de los participantes. } \\
\text { - La estrategia fue implementada logrando altos niveles de confianza } \\
\text { de quienes debieron ejecutar dicha estrategia. } \\
\text { - La estrategia fue implementada logrando altos niveles de armonía } \\
\text { en la institución. } \\
\text { - La estrategia fue implementada logrando altos niveles de obediencia } \\
\text { y cooperación de los encargados de la ejecución. }\end{array}$ & $\begin{array}{l}\text { Basado en } \\
\text { Amason; } \\
\text { Pedraja- } \\
\text { Rejas } \\
\text { Rodríguez- } \\
\text { Ponce } \\
\text { [25 y } 15]\end{array}$ & 0,708 \\
\hline
\end{tabular}




\section{RESULTADOS}

La Tabla 2 muestra las principales estadísticas descriptivas de las variables empleadas.

Tabla 2. Estadísticos descriptivos.

\begin{tabular}{|l|c|c|c|}
\cline { 2 - 4 } \multicolumn{1}{c|}{} & N & Media & Desv. típ. \\
\hline Transforma & 78 & 3,6872 & 1,50276 \\
Transacción & 78 & 3,7981 & 1,44827 \\
Creación & 78 & 3,4795 & 1,37372 \\
Compartir & 78 & 3,6538 & 1,41226 \\
Aplicar & 78 & 3,6667 & 1,70878 \\
Diseño & 78 & 3,7532 & 1,42251 \\
Implementa & 78 & 3,6282 & 1,42436 \\
N válido (según lista) & 78 & & \\
\hline
\end{tabular}

Las medias están cercanas estadísticamente al promedio 3,5 , con máximos que van desde 6 hasta 7 y con mínimos que en todos los casos llegan a 1. Esto implica que hay una gran diversidad de respuestas y que si bien en la muestra los estilos de liderazgo transformacional y transaccional son de nivel medio, existen en la muestra ejecutivos que perciben altos niveles de estos liderazgos y otros que perciben bajos niveles. Igual cosa ocurre con el proceso de crear, compartir y aplicar conocimiento y con el diseño e implementación de la estrategia.

\section{Contrastación de Hipótesis 1:}

Considerando la hipótesis 1 de la investigación, se trabajó con la ecuación de regresión (2).

Diseño de la Estrategia $=A+\beta_{1}$ Liderazgo Transformacional $+\beta_{2}$ Liderazgo Transaccional $+\beta_{3}$ Creación Conocimiento $+\beta_{4}$ Compartir Conocimiento $+\beta_{5}$ Aplicar Conocimiento $+\mathrm{E}_{\mathrm{i}}$

Aplicando esta ecuación de regresión se obtienen los índices de correlación de Pearson mostrados en la Tabla 3.

Tabla 3. Correlación de Pearson.

\begin{tabular}{|c|c|c|c|c|c|c|}
\hline & 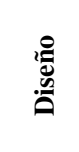 & 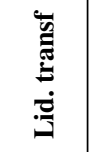 & 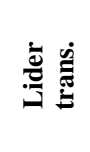 & ن̃ & 竞 & 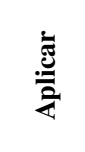 \\
\hline Diseño & 1,000 &, $768 *$ &, $659 *$ & ,723* &, $744 *$ &, $791 *$ \\
\hline Transforma &, 768 & 1,000 & ,650 & ,693 & ,736 & ,760 \\
\hline Transacción & ,659 & ,650 & 1,000 &, 602 &, 545 & ,606 \\
\hline Creación &, 723 & ,693 &, 602 & 1,000 & 693 & ,676 \\
\hline Compartir &, 744 &, 736 &, 545 &, 693 & 1,000 &, 757 \\
\hline Aplicar & ,791 &, 760 & ,606 & ,676 &, 757 & 1,000 \\
\hline
\end{tabular}

* Significancia al $1 \%$.
Las variables liderazgo transformacional y liderazgo transaccional, así como las fases de crear, compartir y aplicar conocimiento se correlacionan de manera significativa con la calidad del diseño de la estrategia $(\mathrm{p}<0.000)$.

La capacidad explicativa del modelo es del 74,1\%, y el análisis de varianza muestra un Test F 41.20, el que es plenamente significativo desde el punto de vista estadístico ( $\mathrm{p}<0,000)$. Los datos anteriores se derivan de las Tablas 4 y 5.

Tabla 4. Resumen del modelo.

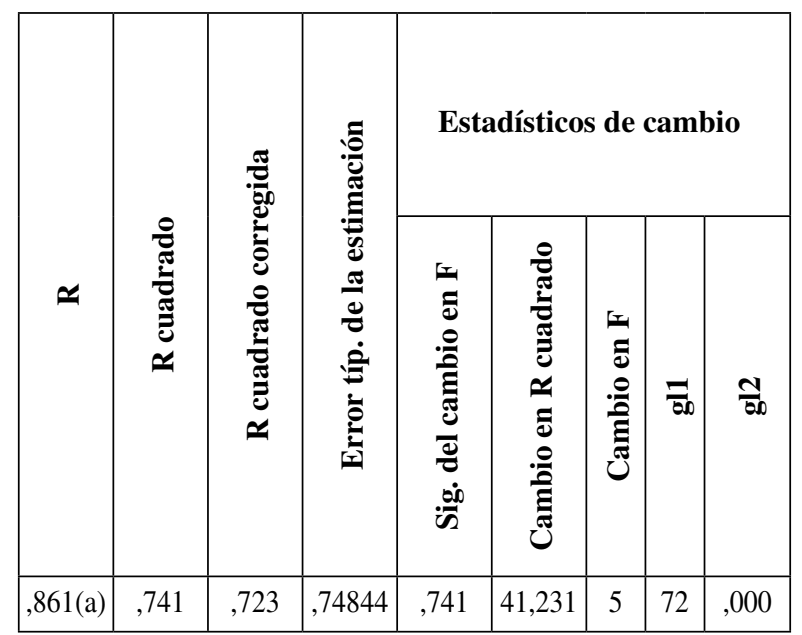

a Variables predictoras: (Constante), aplicar, transacción, creación, compartir, transforma.

Tabla 5. ANOVA (b).

\begin{tabular}{|l|l|c|r|c|c|c|}
\hline \multicolumn{2}{|c|}{ Modelo } & $\begin{array}{c}\text { Suma de } \\
\text { cuadrados }\end{array}$ & gl & $\begin{array}{c}\text { Media } \\
\text { cuadrática }\end{array}$ & F & Sig. \\
\hline 1 & Regresión & 115,480 & 5 & 23,096 & 41,231 &, $000(a)$ \\
& $\begin{array}{l}\text { Residual } \\
\text { Total }\end{array}$ & $\begin{array}{r}40,331 \\
155,812\end{array}$ & 72 &, 560 & & \\
\hline
\end{tabular}

a Variables predictoras: (Constante), aplicar, transacción, creación, compartir, transforma.

b Variable dependiente: diseño.

En consecuencia, el modelo en general tiene una alta capacidad explicativa de la calidad del diseño, lo que supone que los estilos de liderazgo y la gestión del conocimiento son determinantes significativas del diseño de la estrategia.

Ahora bien, al analizar la pertinencia estadística de cada una de las variables del modelo se obtiene que: 1) las variables omitidas no tienen significancia estadística ( $\mathrm{p}<0,219)$; 2) tanto el estilo de liderazgo transformacional ( $p<0,083)$ como el estilo de liderazgo transaccional ( $\mathrm{p}<0,072)$ impactan positivamente sobre la calidad del diseño de la estrategia; 3$)$ el crear $(\mathrm{p}<0,066)$ y aplicar ( $\mathrm{p}<0,004)$ conocimiento son las fases de la gestión del conocimiento que impactan positiva y significativamente 
sobre la calidad del diseño de la estrategia. Estos resultados se muestran en la Tabla 6.

Tabla 6. Coeficientes (a).

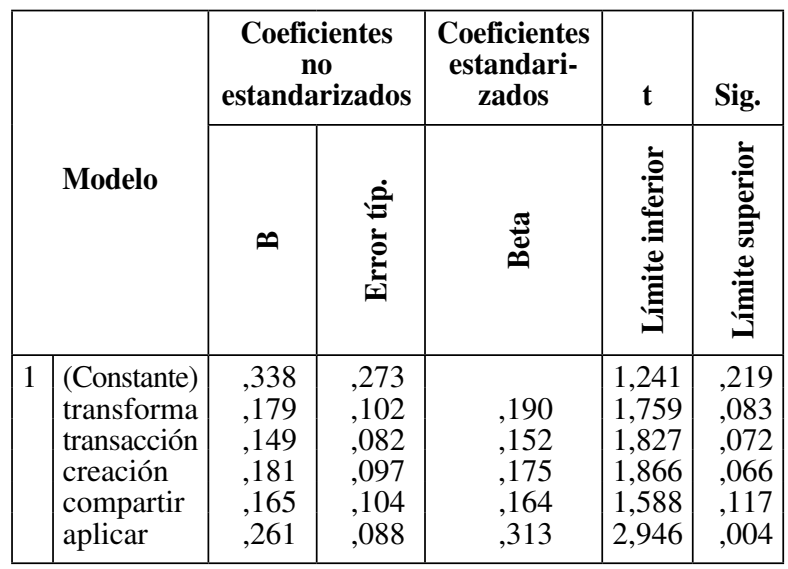

a Variable dependiente: diseño.

\section{Contrastación de Hipótesis 2:}

Considerando la hipótesis 1 de la investigación, se trabajó con la ecuación de regresión (3):

Implementación de la Estrategia $=A+\beta_{1}$ Liderazgo Transformacional $+\beta_{2}$ Liderazgo Transaccional $+\beta_{3}$ Creación Conocimiento $+\beta_{4}$ Compartir Conocimiento + $\beta_{5}$ Aplicar Conocimiento $+\beta_{6}$ Calidad del Diseño $+E_{i}$

Aplicando esta ecuación de regresión se obtienen los siguientes índices de correlación de Pearson, mostrados en la Tabla 7.

Las variables liderazgo transformacional y liderazgo transaccional, así como las fases de crear, compartir y aplicar conocimiento y la calidad del diseño de la estrategia se correlaciona de manera significativa con la calidad de la implementación de la estrategia $(\mathrm{p}<0,000)$.

Tabla 7. Correlación de Pearson.

\begin{tabular}{|c|c|c|c|c|c|c|c|}
\hline & 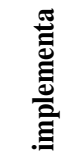 & 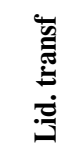 & 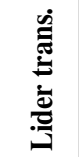 & ن̃ & 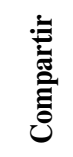 & 苞 & 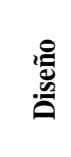 \\
\hline Implementa & 1,000 &, $709 *$ & ,652* & ,704* & ,690* & ,805* &, $860 *$ \\
\hline Transforma & ,709 & 1,000 & ,650 & ,693 & ,736 &, 760 &, 768 \\
\hline Transacción & ,652 & ,650 & 1,000 & ,602 &, 545 & ,606 & 659 \\
\hline Creación & ,704 & ,693 & ,602 & 1,000 & ,693 & ,676 & ,723 \\
\hline Compartir & ,690 & ,736 & ,545 & ,693 & 1,000 & ,757 & ,744 \\
\hline Aplicar & 805 &, 760 & ,606 & ,676 &, 757 & 1,000 & ,791 \\
\hline Diseño &, 860 & ,768 & ,659 & ,723 & ,744 & ,791 & 1,000 \\
\hline
\end{tabular}

* Significancia al $1 \%$.
La capacidad explicativa del modelo es del 77,5\%, y el análisis de varianza muestra un Test F 45.127, el que plenamente significativo desde el punto de vista estadístico $(\mathrm{p}<0,000)$. Los datos anteriores se derivan de las Tablas 8 y 9 .

Tabla 8. Resumen del modelo.

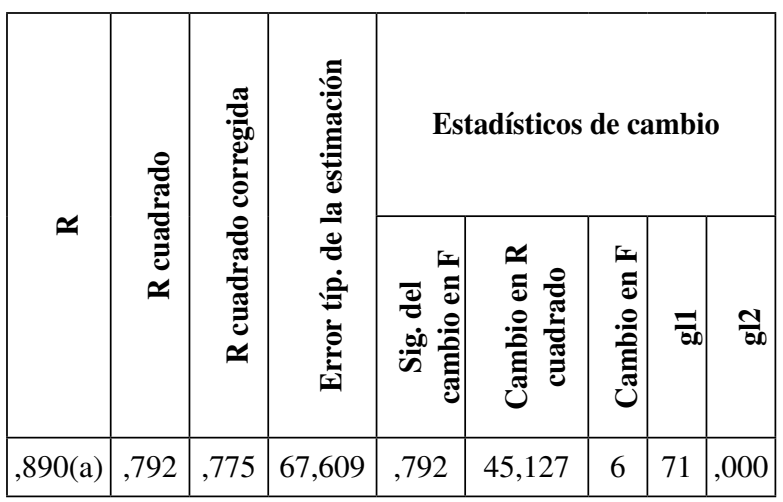

a Variables predictoras: (Constante), aplicar, transacción, creación, compartir, transforma, diseño.

Tabla 9. ANOVA (b).

\begin{tabular}{|c|l|r|r|c|c|c|}
\hline \multicolumn{2}{|c|}{ Modelo } & $\begin{array}{c}\text { Suma de } \\
\text { cuadrados }\end{array}$ & gl & $\begin{array}{c}\text { Media } \\
\text { cuadrática }\end{array}$ & F & Sig. \\
\hline \multirow{2}{*}{1} & Regresión & 115,480 & 5 & 23,096 & 41,231 &, $000(\mathrm{a})$ \\
& Residual & 40,331 & 72 &, 560 & & \\
& Total & 155,812 & 77 & & & \\
\hline
\end{tabular}

a Variables predictoras: (Constante), aplicar, transacción, creación, compartir, transforma, diseño.

b Variable dependiente: implementa.

En consecuencia, el modelo en general tiene una alta capacidad explicativa de la calidad de la implementación de la estrategia, lo que supone que los estilos de liderazgo y la gestión del conocimiento son determinantes significativas del diseño de la estrategia.

Ahora bien, al analizar la pertinencia estadística de cada una de las variables del modelo se obtiene que: 1) las variables omitidas no tienen significancia estadística $(\mathrm{p}<0,382)$; $2)$ tanto el estilo de liderazgo transformacional $(p<0,569)$ como el estilo de liderazgo transaccional $(p<0,209)$ no impactan significativamente sobre la calidad del diseño de la estrategia; 3 ) el aplicar conocimiento $(p<0,002)$ es la fase la gestión del conocimiento que impactan positiva y significativamente sobre la calidad de la implementación de la estrategia; 4) la calidad del diseño de la estrategia es el determinante más importante de la calidad de la implementación de la estrategia $(\mathrm{p}<0,000)$. Estos resultados se muestran en la Tabla 10. 
Tabla 10. Coeficientes (a).

\begin{tabular}{|c|c|c|c|c|c|c|}
\hline & \multirow{2}{*}{ Modelo } & \multicolumn{2}{|c|}{$\begin{array}{c}\text { Coeficientes } \\
\text { no } \\
\text { estandarizados }\end{array}$} & \multirow{2}{*}{$\begin{array}{c}\text { Coeficientes } \\
\text { estandari- } \\
\text { zados }\end{array}$} & \multirow{2}{*}{ 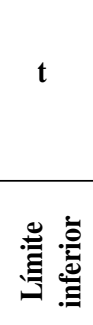 } & \multirow{2}{*}{ 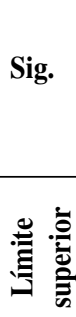 } \\
\hline & & $\varphi$ & 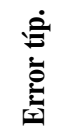 & & & \\
\hline \multirow[t]{7}{*}{1} & (Constante) & ,219 & ,249 & & ,879 & ,382 \\
\hline & transforma &,- 054 & ,094 &,- 057 &,- 573 & ,569 \\
\hline & transacción & ,096 & ,075 & ,097 & 1,267 & ,209 \\
\hline & creación & ,109 & ,090 &, 105 & 1,219 & ,227 \\
\hline & compartir &,- 036 & ,095 &,- 036 &,- 380 & ,705 \\
\hline & aplicar & ,267 & ,085 & ,320 & 3,152 & ,002 \\
\hline & diseño &, 538 & , 106 & ,537 & 5,054 & ,000 \\
\hline
\end{tabular}

a Variable dependiente: implementa.

\section{DISCUSIÓN DE LOS RESULTADOS Y CONCLUSIONES}

Los resultados de la investigación permiten identificar una serie de hallazgos relevantes que pueden ayudar a las pequeñas y medianas empresas a diseñar e implementar mejores estrategias.

Un primer hallazgo dice relación con que los estilos de liderazgo transformacional y transaccional impactan positiva y significativamente sobre la calidad del diseño de la estrategia. Este descubrimiento es consistente con el trabajo de Kavanagh y Ashkanasy [27] quienes enfatizan en la necesidad del ejercicio del liderazgo para tener éxito en las estrategias de cambio organizativo. Más específicamente aún, Vera y Crossan [28] proponen que para lograr éxito estratégico es necesario que ambos estilos transformacional y transaccional sean desarrollados por los líderes, planteamiento plenamente consistente con los hallazgos de esta investigación.

La intuición que subyace en este descubrimiento es que en la medida que exista un liderazgo claro, bien desarrollado, y se perciba dicho liderazgo, este hecho genera un sentido de dirección que ayuda a diseñar de mejor manera la estrategia de la organización.

Un segundo hallazgo relevante dice relación con que la gestión del conocimiento, particularmente la creación y aplicación de conocimiento, es un determinante significativo de la calidad de la estrategia. Este es un descubrimiento que cuenta con evidencia empírica previa [29 y 30]. Sin embargo, vale la pena considerar que en esta investigación es básicamente la creación y la aplicación de conocimientos las que ayudan al diseño de la estrategia; la fase de compartir conocimiento no es relevante desde el punto de vista estadístico. La intuición de este hallazgo está referida a que en un contexto de un claro liderazgo se puede compartir mayor o menor conocimiento, según lo oriente el líder, pero lo esencial es que exista un conocimiento relevante para diseñar la estrategia y que ese conocimiento se aplique efectivamente. Por cierto, el líder permitirá compartir mayores o menores niveles de conocimiento según su estilo, pero lo importante es que ese conocimiento obtenido no se pierda y se aplique aunque sea en forma centralizada y jerárquica.

Un tercer hallazgo, es que el diseño de la estrategia es un determinante clave de la implementación de la estrategia. Este es un hallazgo comúnmente aceptado en la literatura sobre dirección estratégica [31, 32 y 33]; no obstante, de lo anterior se obtiene evidencia de la aplicación de conocimiento para lograr éxito en la implementación de la estrategia. La intuición de este hallazgo sugiere que en la medida que se disponga de suficiente conocimiento para diseñar una buena estrategia, también se estará avanzando en la implementación de la misma. La gestión del conocimientos es por lo tanto importante no sólo para el diseño, sino que también para la implementación de la estrategia.

Finalmente, los hallazgos de esta investigación generan un modelo específico que se presenta en la Figura 2.

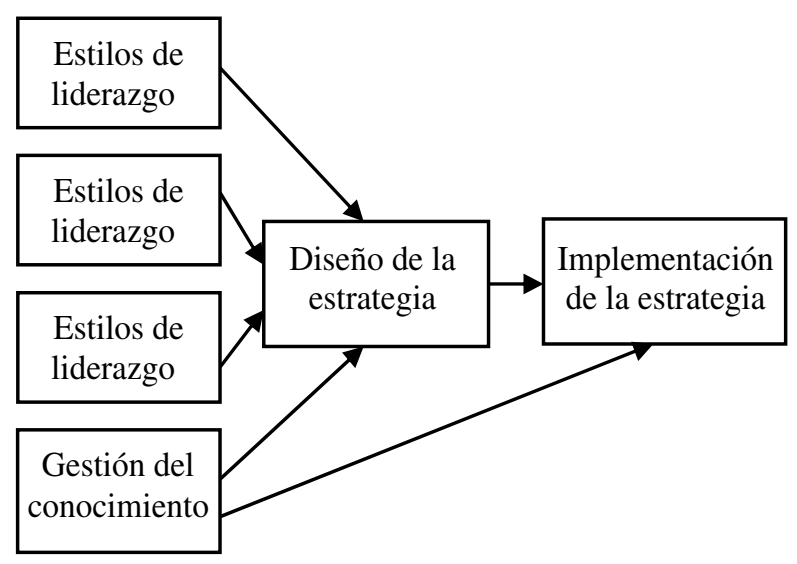

Figura 2. Modelo de análisis.

Este modelo deja en evidencia que tanto los estilos de liderazgo como la gestión del conocimiento son relevantes para lograr mejores niveles de diseño e implementación de la estrategia. En cualquier caso, estos hallazgos deberán ser contrastados en otros contextos que impliquen otros países, tipos de empresas, tamaño de la firma, etc. 
Del mismo modo, en futuras investigaciones se debe estudiar también la etapa de almacenamiento del conocimiento, pues el diseño e implementación de la estrategia pueden ser influenciados por la facilidad de acceder al conocimiento.

\section{REFERENCIAS}

[1] E. Rodríguez-Ponce. "Gestión del conocimiento y eficacia de las organizaciones: Un estudio empírico en instituciones públicas". Interciencia. Vol. 32, pp. 820-826. 2007a.

[2] L. Pedraja-Rejas, E. Rodríguez-Ponce y J. Rodríguez-Ponce. "Sociedad del conocimiento y dirección estratégica una propuesta integradora". Interciencia. Vol. 31, pp. 570-576. 2006a.

[3] E. Rodríguez-Ponce. "La toma de decisiones estratégicas en las instituciones universitarias. Un estudio empírico en Chile y España. Consejo de Rectores de Chile". Andros, 199 p. Santiago, Chile. 2005.

[4] L. Pedraja-Rejas, E. Rodríguez-Ponce y J. RodríguezPonce. "Liderazgo y decisiones estratégicas: Una perspectiva integradora". Interciencia. Vol. 31, pp. $577-582.2006$ b.

[5] K. Cool. "Commentary on procedural justice, strategic decision making and knowledge economy". Strategic Management Journal Vol. 19, pp. 339-341. 1998.

[6] W. Kim and R. Maugborne. "Procedural justice, strategic decision making and the knowledge economy". Strategic Management Journal. Vol. 19, pp. 323-338. 1998.

[7] L. Pedraja-Rejas, E. Rodríguez-Ponce and J. Rodríguez-Ponce. "Leadership styles and effectiveness: a study in small firms en Chile". Interciencia. Vol. 31, pp. 500-504. 2006c.

[8] L. Pedraja-Rejas y E. Rodríguez-Ponce. "Estudio comparativo de la influencia del estilo de liderazgo y congruencia de valores en las empresas privadas e instituciones públicas". Interciencia. Vol. 33, pp. 8-13. 2008.

[9] I. Nonaka and H. Takeuchi. "The KnowledgeCreating Company". Oxford University Press. 284 p. Nueva York, EE.UU. 1995.

[10] G. Hedlung. "A model of knowledge management in n-form corporation". Strategic Management Journal. Vol. 15, pp. 73-90. 1994.

[11] A. Gold, A. Malhotra and A. Segars. "Knowledge management an organizational capabilities perspectives". Journal Management Information System. Vol. 18, pp. 185-214. 2001.

[12] M. Alavi and D. Leidner. "Review: knowledge management and knowledge management systems:
Conceptual foundations and research issues". Management Information System Quarterly. Vol. 25, pp. 107-136. 2001.

[13] G. Hamel and C.K. Prahalad. "Strategy as strecht and leverage". Harvard Business Review. Vol. 71, Issue 2, pp. 75-84. March-April, 1993.

[14] A.A. Thompson, A.J. Strickland and J.E. Gamble. "Strategy: Core Concepts, Analytical Tools Readings". McGraw Hill/Irwin. 512 p. Nueva York, EE.UU. 2005.

[15] L. Pedraja-Rejas y E. Rodríguez-Ponce. "Efectos del estilo de liderazgo sobre la eficacia de las organizaciones públicas". Revista Facultad de Ingeniería, Universidad de Tarapacá. Vol. 12, № 2 , pp. 63-73. 2004.

[16] L. Pedraja-Rejas, E. Rodríguez-Ponce y J. RodríguezPonce. "Liderazgo y decisiones estratégicas: Una perspectiva integradora". Interciencia. Vol. 31, pp. 577-582. 2006b.

[17] S. Rahman. "Total quality management practices and business outcome: Evidence from small and medium enterprises in Western Australia". Total Quality Management. Vol. 12, Issue 2, pp. 201210. 2001.

[18] B. Bass. "From transactional to transformational leadership: Learning to share the vision". Organization Dynamics. Vol. 18, pp. 19-31. 1990.

[19] T. Byers and T. Slack. "Strategic decision-making in small business within the leisure industry". Journal of Leisure Research. Vol. 33, Issue 2, pp. 121-136. 2001.

[20] H. Matlay. "Employee relations in small firms: A micro business perspective". Empl. Relat. Vol. 21, pp. 285-296. 1999.

[21] T. Dean, R. Brown and C. Bamford. "Differences in large and small firm responses to environmental context: Strategic implications from a comparative analysis of business formations". Strategic Management Journal. Vol. 19, pp. 709-728. 1998.

[22] M.J. Chen and D. Hambrick. "Speed, stealth, and selective attack: how small firms differ from large firms in competitive behavior". Academic of Management Journal. Vol. 38, pp. 453-482. 1995.

[23] B.J. Bass and B.M. Avolio. "Improving Organizational Effectiveness Through Transformational Leadership". Sage. Thousand, 248 p. Oaks, CA, EE.UU. 1993.

[24] J.G. March. "Exploration and exploitation in organizational learning". Organization Science. Vol 2, pp. 71-87. February, 1991. 
[25] A.C. Amason. "Distinguishing the effects of functional and dysfunctional conflict on strategic decision making: Resolving a paradox for top management teams". Academic of Management Journal. Vol. 39, Issue 1, pp. 123-148. 1996.

[26] J.W. Dean Jr. and M.P. Sharfman. "Does decision process matter. A study of strategic decision-making effectiveness". Academic of Management Journal. Vol. 39, Issue 2, pp. 368-396. 1996.

[27] M.H. Kavanagh and N.M. Ashkanasy. "The impact of leadership and change management strategy on organizational culture and individual acceptance of change during a merger". British Journal of Management.Vol. 17, pp. 81-103. 2006.

[28] D. Vera and M. Crossan. "Strategic leadership and organizational learning" Academic of Management Review. Vol. 29, Issue 2, pp. 222-240. 2004.

[29] B.D. Janz and P. Prasarnphanich. "Understanding the antecedents of effective knowledge management: the importance of a knowledgecentered culture". Decision Science. Vol. 34, Issue 2, pp. 351-384. 2003.

[30] R. Sabherwal and I. Becerra-Fernández. "An empirical study of the effect of knowledge management processes at individual, group, and organizational levels". Decision Science. Vol. 34, Issue 2, pp. 225-260. 2003.

[31] R. Grant. "Contemporary Strategy Analysis: Concepts, Techniques, Applications". Blackwell Publishing, 548 p. EE.UU. 2005.

[32] D. Lei and J.W. Slocum. "Strategic and organizational requirements for competitive advantage". Academic of Management Executive. Vol. 19, Issue 1, pp. 31- 45. February, 2005.

[33] D.C. Hambrick and J.W. Fredrickson. "Are you sure you have a strategy?". Academic of Management Executive. Vol. 19, Issue 4, pp. 51-62. 2005. 